\title{
Positive periodic solutions for neutral multi-delay logarithmic population model
}

\author{
Mei-Lan Tang ${ }^{*}$ and Xian-Hua Tang
}

\author{
* Correspondence: csutmlang@163. \\ com \\ School of Mathematical Science \\ and Computing Technology, \\ Central South University, Changsha, \\ 410083, China
}

\begin{abstract}
Based on an abstract continuous theorem of $k$-set contractive operator and some analysis skill, a new result is obtained for the existence of positive periodic solutions to a neutral multi-delay logarithmic population model. Some sufficient conditions obtained in this article for the existence of positive periodic solutions to a neutral multi-delay logarithmic population model are easy to check. Furthermore, our main result also weakens the condition in the existing results. An example is used to illustrate the applicability of the main result.
\end{abstract}

MSC 2010: 34C25; 34D40.

Keywords: positive periodic solution, existence, $k$-set contractive operator, logarithmic population model

\section{Introduction}

In recent years, there has been considerable interest in the existence of periodic solutions of functional differential equations (see, for example, [1-7]). It is well known that the environments of most natural populations change with time and that such changes induce variation in the growth characteristics of populations. Among many population models, the neutral logarithmic population model has recently attracted the attention of many mathematicians and biologists.

Let $\omega>0$ be a constant, $C_{\omega}=\{x: x \in C(R, R), x(t+\omega)=x(t)\}$, with the norm defined by $|x|_{0}=\max _{t \in[0, \omega]}|x(t)|$, and $C_{\omega}^{1}=\left\{x: x \in C^{1}(R, R), x(t+\omega)=x(t)\right\}$, with the norm defined by $\|x\|_{0}=\max \left\{|x|_{0},\left|x^{\prime}\right|_{0}\right\}$, then $C_{\omega}, C_{\omega}^{1}$ are both Banach spaces. Let $\bar{h}=\frac{1}{\omega} \int_{0}^{\omega} h(t) d t, \forall h \in C_{\omega}$.

$\mathrm{Lu}$ and Ge [8] studied the existence of positive periodic solutions for neutral logarithmic population model with multiple delays. Based on an abstract continuous theorem of $k$-set contractive operator, Luo and Luo [9] investigate the following periodic neutral multi-delay logarithmic population model:

$$
\frac{d N}{d t}=N(t)\left[r(t)-\sum_{j=1}^{n} a_{j}(t) \ln N\left(t-\sigma_{j}(t)\right)-\sum_{i=1}^{m} b_{i}(t) \frac{d}{d t} \ln N\left(t-\tau_{i}(t)\right)\right]
$$

where $r(t), a_{j}(t), b_{i}(t), \sigma_{j}(t), \tau_{i}(t)$ are all in $C_{\omega}$ with $\bar{r}>0, \sigma_{j}(t)>0$ and $\tau_{i}(t)>0, \forall t \in[0$, $\omega], \forall j \in\{1,2, \ldots, n\}, \forall i \in\{1,2, \ldots, m\}$. Furthermore, $b_{i}(t) \in C^{1}(R, R), \sigma_{j}(t) \in C^{1}(R, R), \tau_{i}(t)$ 
$\in C^{2}(R, R)$ and $\sigma_{j}^{\prime}(t)<1, \tau_{i}^{\prime}(t)<1, \forall j \in\{1,2, \ldots, n\}, \forall i \in\{1,2, \ldots, m\}$.

Since $\sigma_{j}^{\prime}(t)<1, \forall t \in[0, \omega], t-\sigma_{j}(t)$ has a unique inverse. Let $\mu_{j}(t)$ be the inverse of $t-\sigma_{j}(t)$. Similarly, $t-\tau_{i}(t)$ has a unique inverse, denoted by $\gamma_{i}(t)$.

For convenience, denote $\Gamma(t)=\sum_{j=1}^{n} \frac{a_{j}\left(\mu_{j}(t)\right)}{1-\sigma_{j}^{\prime}\left(\mu_{j}(t)\right)}-\sum_{i=1}^{m} \frac{b_{i}^{\prime}\left(\gamma_{i}(t)\right)}{1-\tau_{i}^{\prime}\left(\gamma_{i}(t)\right)}$.

Luo and Luo [9] obtain the following sufficient condition on the existence of positive periodic solutions for neutral logarithmic population model with multiple delays.

Theorem A. Assume the following conditions hold:

$\left(H 1^{\prime}\right)$ There exists a constant $\theta>0$ such that $|\Gamma(t)|>\theta, \forall t \in[0, \omega]$.

$\left(H 2^{\prime}\right) \sum_{j=1}^{n}\left|a_{j}\right|_{0} \omega+\sum_{i=1}^{m}\left|b_{i}\right|_{0}\left|1-\tau_{i}^{\prime}\right|_{0}^{1 / 2}<1$ and $\sum_{i=1}^{m}\left|b_{i}\right|_{0}\left|1-\tau_{i}^{\prime}\right|_{0}<1$.

Then Equation (1) has at least an $\omega$-positive periodic solution.

The purpose of this article is to further consider the existence of positive periodic solutions to a neutral multi-delay logarithmic population model (1). We will present some new sufficient conditions for the existence of positive periodic solutions to a neutral multi-delay logarithmic population model. In this article, we will replace the assumption $\left(H 1^{\prime}\right):|\Gamma(t)|>\theta$ in Theorem A by different assumption $\Gamma(t)>0, \forall t \in[0, \omega],(\operatorname{or} \Gamma(t)<0, \forall t$ $\in[0, \omega])$. Obviously, it is more easy to check $\Gamma(t)>0, \forall t \in[0, \omega]$, than to find a constant $\theta>0$ such that $|\Gamma(t)|>\theta, \forall t \in[0, \omega]$. At the same time, the assumption (H2') in Theorem A will be greatly weakened. $\sum_{j=1}^{n}\left|a_{j}\right|_{0} \omega+\sum_{i=1}^{m}\left|b_{i}\right|_{0}\left|1-\tau_{i}^{\prime}\right|_{0}^{1 / 2}<1$ in Theorem A is replaced by $\frac{1}{2} \sum_{j=1}^{n}\left|a_{j}\right|_{0} \omega+\sum_{i=1}^{m}\left|b_{i}\right|_{0}\left|1-\tau_{i}^{\prime}\right|_{0}^{1 / 2}<1$ in this article.

\section{Main lemmas}

Under the transformation $N(t)=e^{x(t)}$, then Equation (1) can be rewritten in the following form:

$$
x^{\prime}(t)=r(t)-\sum_{j=1}^{n} a_{j}(t) x\left(t-\sigma_{j}(t)\right)-\sum_{i=1}^{m} c_{i}(t) x^{\prime}\left(t-\tau_{i}(t)\right)
$$

where $c_{i}(t)=b_{i}(t)\left(1-\tau_{i}^{\prime}(t)\right), i=1,2, \ldots, m$.

It is easy to see that in this case the existence of positive periodic solution of Equation (1) is equivalent to the existence of periodic solution of Equation (2). In order to investigate the existence of periodic solution of Equation (2), we need some definitions and lemmas.

Definition 1. Let $E$ be a Banach space, $S \subset E$ be a bounded subset, denote $\alpha_{E}(S)=$ $\inf \left\{\delta>0 \mid\right.$ there is a finite number of subsets $S_{i} \subset S$ such that $S=\bigcup_{i} S_{i}$ and

$\left.\operatorname{diamS}_{i} \leq \delta\right\}$

then $\alpha_{E}$ is called non-compactness measure of $S$ or Kuratowski distance (see [1]), where diamS $S_{i}$ denotes the diameter of set $S_{i}$.

Definition 2. Let $E_{1}$ and $E_{2}$ be Banach spaces, $D \subset E_{1}, A: D \rightarrow E_{2}$ be a continuous and bounded operator. If there exists a constant $k \geq 0$ satisfying $\alpha_{E_{2}}(A(S)) \leq k \alpha_{E_{1}}(S)$ for any bounded set $S \subset D$, then $A$ is called $k$-set contractive operator on $D$.

Definition 3. Let $X, Y$ be normed vector spaces, $L: D o m L \subset X \rightarrow Y$ be a linear mapping. This mapping $L$ will be called a Fredholm mapping of index 0 if $\operatorname{dimKerL}=$ codi$m \operatorname{ImL}<\infty$ and ImL is closed in $Y$ [3]. 
Assume that $L: D o m L \subset X \rightarrow Y$ is a Fredholm operator with index 0, from [3], we know that $\sup \left\{\delta>0 \mid \delta \alpha_{X}(B) \leq \alpha_{Y}(L(B))\right\}$ exists for any bounded set $B \subset D o m L$, so we can define

$$
l(L)=\sup \left\{\delta>0 \mid \delta \alpha_{X}(B) \leq \alpha_{Y}(L(B)) \text { for any bounded set } B \subset D o m L\right\} .
$$

Now let $L: X \rightarrow Y$ be a Fredholm operator with index $0, X$ and $Y$ be Banach spaces, $\Omega \subset X$ be an open and bounded set, and let $N: \bar{\Omega} \rightarrow Y$ be a $k$-set contractive operator with $k<l(L)$. By using the homotopy invariance of $k$-set contractive operator's topological degree $D[(L, N), \Omega]$, Petryshyn and $\mathrm{Yu}[10]$ proved the following result.

Lemma 1. [10]Assume that $L: X \rightarrow Y$ is a Fredholm operator with index $0, r \in Y$ is a fixed point, $N: \bar{\Omega} \rightarrow Y$ is a k-set contractive operator with $k<l(L)$, where $\Omega \subset X$ is bounded, open, and symmetric about $0 \in \Omega$. Furthermore, we also assume that

(R1) $L x \neq \lambda N x+\lambda r, \forall \lambda \in(0,1), \quad \forall x \in \partial \Omega \cap D o m L ;$

$(R 2)[Q N(x)+Q r, x][Q N(-x)+Q r, x]<0, \quad \forall x \in \partial \Omega \cap \operatorname{KerL}$.

where[,., $]$ is a bilinear form on $Y \times X$, and $Q$ is the projection of $Y$ onto Coker, where Coker is the cokernel of the operator $L$. Then there exists a $x \in \bar{\Omega}$ satisfying $L x=N x+r$.

In the rest of this article, we set $Y=C_{\omega}, X=C_{\omega}^{1}$

$$
L x=\frac{d x}{d t}
$$

and

$$
N x=-\sum_{j=1}^{n} a_{j}(t) x\left(t-\sigma_{j}(t)\right)-\sum_{i=1}^{m} c_{i}(t) x^{\prime}\left(t-\tau_{i}(t)\right),
$$

then Equation (2) is equivalent to the equation

$$
L x=N x+r
$$

where $r=r(t)$. Clearly, Equation (2) has an $\omega$-periodic solution if and only if Equation (5) has a solution $x \in C_{\omega}^{1}$.

Lemma 2. [7] The differential operator $L$ is a Fredholm operator with index 0 , and satisfies $l(L) \geq 1$.

Lemma 3. [9]If $k=\sum_{i=1}^{n}\left|c_{i}\right|_{0}$, then $N: \Omega \rightarrow C_{\omega}$ is a $k$-set contractive operator.

Lemma 4. [8] Suppose $\tau \in C_{\omega}^{1}$ and $\tau^{\prime}(t)<1, \forall t \in[0, \omega]$. Then the function $t-\tau(t)$ has a inverse $\mu(t)$ satisfying $\mu \in C(R, R)$ with $\mu(a+\omega)=\mu(a)+\omega$.

Lemma 5. [11] Let $x(t)$ be continuous differentiable T-periodic function $(T>0)$. Then for any $t_{*} \in(-\infty,+\infty)$

$$
\max _{t \in\left[t_{*}, t_{*}+T\right]}|x(t)| \leq\left|x\left(t_{*}\right)\right|+\frac{1}{2} \int_{0}^{T}\left|x^{\prime}(s)\right| d s .
$$

\section{Main results}

Let $\mu_{j}(t)$ be the inverse of $t-\sigma_{j}(t), \gamma_{j}(t)$ be the inverse of $t-\tau_{i}(t)$ and $\Gamma(t)=\sum_{j=1}^{n} \frac{a_{j}\left(\mu_{j}(t)\right)}{1-\sigma_{j}^{\prime}\left(\mu_{j}(t)\right)}-\sum_{i=1}^{m} \frac{b_{i}^{\prime}\left(\gamma_{i}(t)\right)}{1-\tau_{i}^{\prime}\left(\gamma_{i}(t)\right)}$. 
Theorem 1. Assume the following conditions hold:

(H1) If $\Gamma(t)>0, \forall t \in[0, \omega]($ or $\Gamma(t)<0, \forall t \in[0, \omega])$;

(H2) $\frac{1}{2} \sum_{j=1}^{n}\left|a_{j}\right|_{0} \omega+\sum_{i=1}^{m}\left|b_{i}\right|_{0}\left|1-\tau_{i}^{\prime}\right|_{0}^{1 / 2}<1$ and $\sum_{i=1}^{m}\left|b_{i}\right|_{0}\left|1-\tau_{i}^{\prime}\right|_{0}<1$.

Then Equation (1) has at least an w-positive periodic solution.

Proof. Suppose that $x(t)$ is an arbitrary $\omega$-periodic solution of the following operator equation

$$
L x=\lambda N x+\lambda r
$$

where $L$ and $N$ are defined by Equations (3) and (4), respectively. Then $x(t)$ satisfies

$$
x^{\prime}(t)=\lambda\left[r(t)-\sum_{j=1}^{n} a_{j}(t) x\left(t-\sigma_{j}(t)\right)-\sum_{i=1}^{m} c_{i}(t) x^{\prime}\left(t-\tau_{i}(t)\right)\right] .
$$

Integrating both sides of Equation (7) over $[0, \omega]$ gives

$$
\int_{0}^{\omega}\left[r(t)-\sum_{j=1}^{n} a_{j}(t) x\left(t-\sigma_{j}(t)\right)+\sum_{i=1}^{m} b_{i}^{\prime}(t) x\left(t-\tau_{i}(t)\right)\right] d t=0
$$

i.e.,

$$
\int_{0}^{\omega}\left[\sum_{j=1}^{n} a_{j}(t) x\left(t-\sigma_{j}(t)\right)-\sum_{i=1}^{m} b_{i}^{\prime}(t) x\left(t-\tau_{i}(t)\right)\right] d t=\bar{r} \omega .
$$

Let $t-\sigma_{j}(t)=s$, i.e., $t=\mu_{j}(s)$. Lemma 4 implies that

$$
\frac{a_{j}\left(\mu_{j}(s)\right)}{1-\sigma_{j}^{\prime}\left(\mu_{j}(s)\right)} \in C_{\omega}, \quad \frac{a_{j}\left(\mu_{j}(s)\right)}{1-\sigma_{j}^{\prime}\left(\mu_{j}(s)\right)} x(s) \in C_{\omega} .
$$

Lemma 4 implies $\mu_{j}(0+\omega)=\mu_{j}(0)+\omega, \gamma_{i}(0+\omega)=\gamma_{i}(0)+\omega, \forall j \in\{1, \ldots, n\}, i \in\{1, \ldots, m\}$. Noting that $\sigma_{j}(0)=\sigma_{j}(\omega), \tau_{i}(0)=\tau_{i}(\omega)$, then

$$
\begin{aligned}
& \int_{0}^{\omega} \frac{a_{j}\left(\mu_{j}(s)\right)}{1-\sigma_{j}^{\prime}\left(\mu_{j}(s)\right)} d s=\int_{-\sigma_{j}(0)}^{\omega-\sigma_{j}(\omega)} \frac{a_{j}\left(\mu_{j}(s)\right)}{1-\sigma_{j}^{\prime}\left(\mu_{j}(s)\right)} d s=\int_{0}^{\omega} a_{j}(t) d t=\omega \bar{a}_{j,} \quad j=1, \ldots, n, \\
& \int_{0}^{\omega} \frac{b_{i}^{\prime}\left(\gamma_{i}(s)\right)}{1-\tau_{i}^{\prime}\left(\gamma_{i}(s)\right)} d s=\int_{-\tau_{i}(0)}^{\omega-\tau_{i}(\omega)} \frac{b_{i}^{\prime}\left(\gamma_{i}(s)\right)}{1-\tau_{i}^{\prime}\left(\gamma_{i}(s)\right)} d s=\int_{0}^{\omega} b_{i}^{\prime}(t) d t=0, \quad i=1, \ldots, m .
\end{aligned}
$$

Noting that $\Gamma(t)>0$, we have

$$
\bar{\Gamma}=\frac{1}{\omega} \int_{0}^{\omega} \Gamma(t) d t=\frac{1}{\omega} \int_{0}^{\omega}\left[\sum_{j=1}^{n} \frac{a_{j}\left(\mu_{j}(t)\right)}{1-\sigma_{j}^{\prime}\left(\mu_{j}(t)\right)}-\sum_{i=1}^{m} \frac{b_{i}^{\prime}\left(\gamma_{i}(t)\right)}{1-\tau_{i}^{\prime}\left(\gamma_{i}(t)\right)}\right] d t=\sum_{j=1}^{n} \bar{a}_{j}>0 .
$$

Furthermore

$$
\begin{aligned}
\int_{0}^{\omega} a_{j}(t) x\left(t-\sigma_{j}(t)\right) d t & =\int_{-\sigma_{j}(0)}^{\omega-\sigma_{j}(\omega)} \frac{a_{j}\left(\mu_{j}(s)\right)}{1-\sigma_{j}^{\prime}\left(\mu_{j}(s)\right)} x(s) d s \\
& =\int_{0}^{\omega} \frac{a_{j}\left(\mu_{j}(s)\right)}{1-\sigma_{j}^{\prime}\left(\mu_{j}(s)\right)} x(s) d s, \quad j=1, \ldots, n .
\end{aligned}
$$


Similarly

$$
\begin{aligned}
\int_{0}^{\omega} b_{i}^{\prime}(t) x\left(t-\tau_{i}(t)\right) d t & =\int_{-\tau_{i}(0)}^{\omega-\tau_{i}(\omega)} \frac{b_{i}^{\prime}\left(\gamma_{i}(s)\right)}{1-\tau_{i}^{\prime}\left(\gamma_{i}(s)\right)} x(s) d s \\
& =\int_{0}^{\omega} \frac{b_{i}^{\prime}\left(\gamma_{i}(s)\right)}{1-\tau_{i}^{\prime}\left(\gamma_{i}(s)\right)} x(s) d s, \quad i=1, \ldots, m .
\end{aligned}
$$

Combining (13) and (14) with (9) yields

$$
\int_{0}^{\omega} \Gamma(t) x(t) d t=\bar{r} \omega .
$$

Since $\Gamma(t)>0$, it follows from the extended integral mean value theorem that there exists $\eta \in[0, \omega]$ satisfying

$$
x(\eta) \int_{0}^{\omega} \Gamma(t) d t=\bar{r} \omega,
$$

i.e.,

$$
x(\eta)=\frac{\bar{r}}{\bar{\Gamma}} .
$$

By Lemma 5, we obtain

$$
|x(t)| \leq|x(\eta)|+\frac{1}{2} \int_{0}^{\omega}\left|x^{\prime}(t)\right| d t .
$$

So

$$
|x|_{0} \leq\left|\frac{\bar{r}}{\bar{\Gamma}}\right|+\frac{1}{2} \int_{0}^{\omega}\left|x^{\prime}(t)\right| d t .
$$

Multiplying both sides of Equation (7) by $x^{\prime}(t)$ and integrating them over $[0, \omega]$, we have

$$
\begin{aligned}
& \int_{0}^{\omega}\left|x^{\prime}(t)\right|^{2} d t \\
= & \int_{0}^{\omega} x^{\prime}(t)^{2} d t \\
= & \left|\int_{0}^{\omega} x^{\prime}(t)^{2} d t\right| \\
= & \lambda\left|\int_{0}^{\omega} r(t) x^{\prime}(t) d t-\int_{0}^{\omega} \sum_{j=1}^{n} a_{j}(t) x\left(t-\sigma_{j}(t)\right) x^{\prime}(t) d t-\int_{0}^{\omega} \sum_{i=1}^{m} c_{i}(t) x^{\prime}\left(t-\tau_{i}(t)\right) x^{\prime}(t) d t\right| \\
\leq & |r|_{0} \int_{0}^{\omega}\left|x^{\prime}(t)\right| d t+\sum_{j=1}^{n}\left|a_{j}\right|_{0}|x|_{0} \int_{0}^{\omega}\left|x^{\prime}(t)\right| d t+\sum_{i=1}^{m} \int_{0}^{\omega}\left|c_{i}(t) x^{\prime}\left(t-\tau_{i}(t)\right) x^{\prime}(t)\right| d t .
\end{aligned}
$$


Cauchy-Schwarz inequality implies

$$
\begin{aligned}
\int_{0}^{\omega}\left|x^{\prime}(t)\right|^{2} d t= & \lambda \mid \int_{0}^{\omega} r(t) x^{\prime}(t) d t-\int_{0}^{\omega} \sum_{j=1}^{n} a_{j}(t) x\left(t-\sigma_{j}(t)\right) x^{\prime}(t) d t \\
& -\int_{0}^{\omega} \sum_{i=1}^{m} c_{i}(t) x^{\prime}\left(t-\tau_{i}(t)\right) x^{\prime}(t) d t \mid \\
\leq & \left.|r|_{0}+\sum_{j=1}^{n}\left|a_{j}\right|_{0}|x|_{0}\right)\left(\int_{0}^{\omega}\left|x^{\prime}(t)\right|^{2} d t\right)^{1 / 2} \omega^{1 / 2}+ \\
& \sum_{i=1}^{m}\left(\int_{0}^{\omega}\left|c_{i}(t) x^{\prime}\left(t-\tau_{i}(t)\right)\right|^{2} d t\right)^{1 / 2}\left(\int_{0}^{\omega}\left|x^{\prime}(t)\right|^{2} d t\right)^{1 / 2} .
\end{aligned}
$$

Meanwhile

$$
\begin{aligned}
\left(\int_{0}^{\omega}\left|c_{i}(t) x^{\prime}\left(t-\tau_{i}(t)\right)\right|^{2} d t\right)^{1 / 2} & =\left(\int_{0}^{\omega} \frac{1}{1-\tau_{i}^{\prime}\left(\gamma_{i}(t)\right)}\left|c_{i}\left(\gamma_{i}(t)\right) x^{\prime}(t)\right|^{2} d t\right)^{1 / 2} \\
& =\left(\int_{0}^{\omega}\left(1-\tau_{i}^{\prime}\left(\gamma_{i}(t)\right)\right)\left|b_{i}\left(\gamma_{i}(t)\right) x^{\prime}(t)\right|^{2} d t\right)^{1 / 2} \\
& \leq\left|1-\tau_{i}^{\prime}\right|_{0}^{1 / 2}\left|b_{i}\right|_{0}\left(\int_{0}^{\omega}\left|x^{\prime}(t)\right|^{2} d t\right)^{1 / 2} .
\end{aligned}
$$

Substituting Equations (18) and (21) into (20) gives

$$
\begin{aligned}
\int_{0}^{\omega}\left|x^{\prime}(t)\right|^{2} d t \leq & \left(|r|_{0}+\sum_{j=1}^{n}\left|a_{j}\right|_{0}|x|_{0}\right)\left(\int_{0}^{\omega}\left|x^{\prime}(t)\right|^{2} d t\right)^{1 / 2} \omega^{1 / 2} \\
& +\sum_{i=1}^{m}\left|1-\tau_{i}^{\prime}\right|_{0}^{1 / 2}\left|b_{i}\right|_{0}\left(\int_{0}^{\omega}\left|x^{\prime}(t)\right|^{2} d t\right) \\
\leq & \left(|r|_{0}+\sum_{j=1}^{n}\left|a_{j}\right|_{0} \frac{\bar{r}}{\bar{\Gamma}}\right)\left(\int_{0}^{\omega}\left|x^{\prime}(t)\right|^{2} d t\right)^{1 / 2} \omega^{1 / 2}+ \\
& {\left[\frac{1}{2} \omega \sum_{j=1}^{n}\left|a_{j}\right|_{0}+\sum_{i=1}^{m}\left|1-\tau_{i}^{\prime}\right|_{0}^{1 / 2}\left|b_{i}\right|_{0}\right]\left(\int_{0}^{\omega}\left|x^{\prime}(t)\right|^{2} d t\right) . }
\end{aligned}
$$

From the assumption $\frac{1}{2} \omega \sum_{j=1}^{n}\left|a_{j}\right|_{0}+\sum_{i=1}^{m}\left|1-\tau_{i}^{\prime}\right|_{0}^{1 / 2}\left|b_{i}\right|_{0}<1$, it follows from Equation (22) that there exists constant $M>0$ such that

$$
\left(\int_{0}^{\omega}\left|x^{\prime}(t)\right|^{2} d t\right)^{1 / 2}<M .
$$


Then

$$
|x|_{0} \leq\left|\frac{\bar{r}}{\bar{\Gamma}}\right|+\frac{1}{2} \int_{0}^{\omega}\left|x^{\prime}(t)\right| d t \leq\left|\frac{\bar{r}}{\bar{\Gamma}}\right|+\frac{1}{2} M \omega^{1 / 2}:=M_{1} .
$$

Again from (7), we get

$$
\left|x^{\prime}\right|_{0} \leq|r|_{0}+\sum_{j=1}^{n}\left|a_{j}\right|_{0}|x|_{0}+\sum_{i=1}^{m}\left|c_{i}\right|_{0}\left|x^{\prime}\right|_{0} .
$$

Condition $\sum_{i=1}^{m}\left|c_{i}\right|_{0} \leq \sum_{i=1}^{m}\left|b_{i}\right|_{0}\left|1-\tau_{i}^{\prime}\right|_{0}<1$ implies that

$$
\left|x^{\prime}\right|_{0} \leq \frac{|r|_{0}+\sum_{j=1}^{n}\left|a_{j}\right|_{0} M_{1}}{1-\sum_{i=1}^{m}\left|c_{i}\right|_{0}}:=M_{2} \text {. }
$$

Let $\quad M_{3}>\max \left\{M_{1}, M_{2},\left|\bar{r} / \sum_{j=1}^{n} \bar{a}_{j}\right|\right\} \quad$ and $\quad \Omega=\left\{x \mid x \in C_{\omega^{\prime}}^{1}\|x\|<M_{3}\right\}$. Then $k=\sum_{i=1}^{m}\left|c_{i}\right|_{0}<1 \leq l(L)$. Equations (24) and (26) imply that all conditions of Lemma 1 except (R2) hold. Next, we prove that the condition (R2) of Lemma 1 is also satisfied. We define a bounded bilinear form $[\cdot, \cdot]$ on $C_{\omega} \times C_{\omega}^{1}$ as follows:

$$
[y, x]=\int_{0}^{\omega} \gamma(t) x(t) d t
$$

Define $Q: Y \rightarrow$ CokerL by

$$
Q \gamma=\frac{1}{\omega} \int_{0}^{\omega} \gamma(t) d t
$$

Obviously,

$$
\{x \mid x \in \operatorname{kerL} \bigcap \partial \Omega\}=\left\{x \mid x=M_{3}, x=-M_{3}\right\}
$$

Without loss of generality, we may assume that $x=M_{3}$. Thus

$$
\begin{aligned}
& {[Q N(x)+Q r, x][Q N(-x)+Q r, x] } \\
= & M_{3}^{2}\left[\int_{0}^{\omega} r(t) d t-M_{3} \sum_{j=1}^{n} \int_{0}^{\omega} a_{j}(t) d t\right]\left[\int_{0}^{\omega} r(t) d t+M_{3} \sum_{j=1}^{n} \int_{0}^{\omega} a_{j}(t) d t\right] \\
= & \omega^{2} M_{3}^{2}\left[\bar{r}-M_{3} \sum_{j=1}^{n} \bar{a}_{j}\right]\left[\bar{r}+M_{3} \sum_{j=1}^{n} \bar{a}_{j}\right] \\
< & 0 .
\end{aligned}
$$

Therefore, by Lemma 1, Equation (1) has at least an $\omega$-positive periodic solution.

$$
\text { Since } \quad\left|1-\tau_{i}^{\prime}\right|_{0}<1, \quad \text { then } \quad\left|1-\tau_{i}^{\prime}\right|_{0}<\left|1-\tau_{i}^{\prime}\right|_{0}^{1 / 2} \text {. So }
$$
$\sum_{i=1}^{m}\left|b_{i}\right|_{0}\left|1-\tau_{i}^{\prime}\right|_{0}<\sum_{i=1}^{m}\left|b_{i}\right|_{0}\left|1-\tau_{i}^{\prime}\right|_{0}^{1 / 2}$. From Theorem 1, we have

Corollary 1. Assume that the following conditions hold

$\left(H 1^{\prime}\right)$ If $\Gamma(t)>0, \forall t \in[0, \omega]($ or $\Gamma(t)<0, \quad \forall t \in[0, \omega])$. 


$$
\left(H 2^{\prime}\right) \frac{1}{2} \sum_{j=1}^{n}\left|a_{j}\right|_{0} \omega+\sum_{i=1}^{m}\left|b_{i}\right|_{0}\left|1-\tau_{i}^{\prime}\right|_{0}^{1 / 2}<1 \text { and }\left|1-\tau_{i}^{\prime}\right|_{0}<1, i=1, \ldots, m \text {. }
$$

Then Equation (1) has at least an w-positive periodic solution.

\section{Example}

Example 1 is given to illustrate the effectiveness of our new sufficient conditions, also to demonstrate the difference between the proposed result in this paper and the result in [9].

Example 1. Consider the following equation:

$$
\frac{d N}{d t}=N(t)\left[r(t)-\frac{1}{8}\left(\cos ^{2} t+1\right) \ln N(t-\pi)-\frac{1}{64}(3-\cos t) \frac{d}{d t} \ln N(t-\pi)\right]
$$

where $r(t)=\cos t-\frac{1}{32}\left(\cos ^{2} t+1\right) \sin t-\frac{1}{64}(3-\cos t) \cos t$.

Let $\omega=2 \pi$. Corresponding to Equation (1), we have $n=m=1, a_{1}(t)=\frac{1}{8}\left(\cos ^{2} t+1\right)$,

$$
\begin{aligned}
b_{1}(t)= & \frac{1}{64}(3-\cos t), \quad \sigma_{1}(\mathrm{t})=\tau_{1}(t)=\pi \quad \text { So } \quad \bar{r}=\frac{1}{128}>0, \\
\sigma_{1}^{\prime}(t)= & \tau_{1}^{\prime}(t)=0, \mu_{1}(t)=\gamma_{1}(t)=\pi+t . \text { Thus } \\
& \Gamma(t)=a_{1}\left(\mu_{1}(t)\right)-b_{1}^{\prime}\left(\gamma_{1}(t)\right)=\frac{1}{8}\left(\cos ^{2} t+1\right)+\frac{1}{64} \sin t>0, \\
& \frac{1}{2}\left|a_{1}\right|_{0} \omega+\left|b_{1}\right|_{0}\left|1-\tau_{i}^{\prime}\right|_{0}^{1 / 2}=\frac{4 \pi+1}{16}<1 .
\end{aligned}
$$

The conditions in Theorem 1 in this article are satisfied. Hence Equation (29) has at least an $2 \pi$-positive periodic solution. However, the condition $\left(H_{2}^{\prime}\right)$ in Theorem A(Theorem 3.1 in [9]) is not satisfied. Since

$$
\left|a_{1}\right|_{0} \omega+\left|b_{1}\right|_{0}\left|1-\tau_{i}^{\prime}\right|_{0}^{1 / 2}=\frac{8 \pi+1}{16}>1,
$$

Theorem 3.1 in [9] can not be applied to this example. Let $\theta=\frac{7}{64}$. Although the condition $\left(H_{1}^{\prime}\right)$ in Theorem A (Theorem 3.1 in [9]) is satisfied, it is more complex to check the condition $|\Gamma(t)|>\theta, \forall t \in[0, \omega]$ in Theorem $A$ than to test $\Gamma(t)>0, \forall t \in[0, \omega]$. This example illustrates the advantages of the proposed results in this paper over the existing ones.

\section{Acknowledgements}

The authors are grateful to the referees for their valuable comments which have led to improvement of the presentation. This study was partly supported by the Zhong Nan Da Xue Qian Yan Yan Jiu Ji Hua under grant No. 2010QZZD015, Hunan Scientific Plan under grant No. 2011FJ6037, NSFC under grant No. 61070190 and NFSS under grant 10BJL020.

\section{Authors' contributions}

All authors contributed equally to the manuscript and read and approved the final manuscript.

\section{Competing interests}

The authors declare that they have no competing interests.

Received: 7 March 2011 Accepted: 16 January 2012 Published: 16 January 2012

\section{References}

1. Deimling, K: Nonlinear Functional Analysis. Springer, Berlin (1985)

2. Gopalsamy, K, He, X, Wen, L: On a periodic neutral logistic equation. Glasgow Math J. 33, 281-286 (1991). doi:10.1017/ S001708950000834X

3. Gaines, RE, Mawhin, JL: Coincidence degree and nonlinear differential equation. In Lecture notes in Math, vol. 568, Springer, Berlin (1997) 
4. Kirlinger, G: Permanence in Lotka-Volterra equation, linked prey-predator system. Math Biosci. 82, 165-191 (1986). doi:10.1016/0025-5564(86)90136-7

5. Kuang, Y: Delay Differential Equations with Applications in Population Dynamics. Academic Press, New York (1993)

6. Kuang, Y, Feldstein, A: Boundedness of a nonlinear nonautonomous neutral delay equation. J Math Anal Appl. 156, 293-304 (1991). doi:10.1016/0022-247X(91)90398-J

7. Liu, ZD, Mao, YP: Existence theorem for periodic solutions of higher order nonlinear differential equations. J Math Anal Appl. 216, 481-490 (1997). doi:10.1006/jmaa.1997.5669

8. Lu, SP, Ge, WG: Existence of positive periodic solutions for neutral logarithmic population model with multiple delays. J Comput Appl Math. 166(2), 371-383 (2004). doi:10.1016/j.cam.2003.08.033

9. Luo, Y, Luo, ZG: Existence of positive periodic solutions for neutral multi-delay logarithmic population model. Appl Math Comput. 216, 1310-1315 (2010). doi:10.1016/j.amc.2010.02.024

10. Petryshyn, W, Yu, ZS: Existence theorems for higher order nonlinear periodic boundary value problems. Nonlinear Anal. 9, 943-969 (1982)

11. Tang, ML, Liu, XG, Liu, XB: New results on periodic solution for a kind of Rayleigh equation. Appl Math. 54, 79-85 (2009). doi:10.1007/s10492-009-0006-8

doi:10.1186/1029-242X-2012-10

Cite this article as: Tang and Tang: Positive periodic solutions for neutral multi-delay logarithmic population model. Journal of Inequalities and Applications 2012 2012:10.

\section{Submit your manuscript to a SpringerOpen ${ }^{\odot}$} journal and benefit from:

- Convenient online submission

- Rigorous peer review

- Immediate publication on acceptance

- Open access: articles freely available online

- High visibility within the field

- Retaining the copyright to your article

Submit your next manuscript at $\gg$ springeropen.com 